Check for updates

Cite this: Mater. Adv., 2021, 2, 7366

Received 5th August 2021,

Accepted 3rd October 2021

DOI: $10.1039 / d 1 m a 00693 b$

rsc.li/materials-advances

\section{Synthesis of ultrafine polymer nanofibers $\dagger$}

\author{
Hongyu Meng (D) *abc and Xuezhi Qiao (D) ${ }^{c}$
}

The preparation of nanofibers with finer diameters is of great importance to meet the needs of nanomaterials in fabricating nano-devices. The diameter of polydivinylbenzene nanofibers can be reduced from $200 \mathrm{~nm}$ to $20-50 \mathrm{~nm}$ by adding a very small amount of oleic acid-capped $\mathrm{Fe}_{3} \mathrm{O}_{4}$ nanoparticles during the polymerization of divinylbenzene. Through ultrasonic disruption and magnetic separation of the nanofiber composites, we can obtain purified ultrafine nanofibers without $\mathrm{Fe}_{3} \mathrm{O}_{4}$ nanoparticles. The ultrafine nanofibers have widespread potential applications in nanomaterials and nano-devices with smaller requirements.

One-dimensional (1D) nanomaterials have aroused great interest in both fundamental research and practical applications. ${ }^{1}$ For example, carbon nanofibers and nanotubes have demonstrated potential applications in microelectronics, ${ }^{2,3}$ imaging and therapeutics, ${ }^{4}$ and as adsorbents for organic solvents ${ }^{5}$ and heavy metal ions ${ }^{6}$ in addition, polyaniline nanofibers can be used as conductive nano-devices. ${ }^{7}$ In order to meet the needs of nanomaterials in fabricating small devices, preparation of nanofibers with finer diameters is of great importance.

We have previously reported a method for the large-scale synthesis of crosslinked polydivinylbenzene (PDVB) nanofibers via template-free rapid cationic polymerization. ${ }^{8}$ The initiator boron trifluoride diethyl etherate (BFEE) is dispersed in $n$-hexane as immiscible nano-droplets under ultrasonication. When the monomer divinylbenzene (DVB) is introduced, the cationic polymerization of DVB occurs on the surface of the BFEE droplets, forming crosslinked PDVB. Eventually the PDVB nanofibers, with a diameter of

\footnotetext{
${ }^{a}$ CAS Center for Excellence in Nanoscience, Beijing Key Laboratory of Micro-Nano Energy and Sensor, Beijing Institute of Nanoenergy and Nanosystems, Chinese Academy of Sciences, Beijing, 100083, China. E-mail: menghongyu@binn.cas.cn

${ }^{b}$ School of Nanoscience and Technology, University of Chinese Academy of Sciences, Beijing 100049, China

${ }^{c}$ Beijing National Laboratory for Molecular Sciences, State Key Laboratory of Polymer Physics and Chemistry, Key Laboratory of Analytical Chemistry for Living Biosystems, Institute of Chemistry, Chinese Academy of Sciences (CAS),

Beijing 100190, China

$\dagger$ Electronic supplementary information (ESI) available. See DOI: 10.1039/d1ma00693b
}

about $200 \mathrm{~nm}$ (Fig. 2a and b), were formed upon removal of the droplets. Compared with carbon nanofibers and polyaniline nanofibers, PDVB nanofibers have abundant reactive groups ${ }^{8}$ and their morphology and monomer component is variable, ${ }^{9,10}$ which has great advantages in their further modification for use in nanomaterials and nano-devices. ${ }^{11}$ Furthermore, compared with other methods of preparing nanofibers, including electro-spinning ${ }^{12,13}$ and the template method, ${ }^{14,15}$ our method has the advantages of low cost, high efficiency and ease of large-scale preparation.

Herein, we develop an approach to prepare ultrafine PDVB nanofibers with a diameter of 20-50 $\mathrm{nm}$. In the presence of oleic acid-capped $\mathrm{Fe}_{3} \mathrm{O}_{4}$ nanoparticles (NPs) (Fig. S1, ESI $\dagger$ ), ultrafine PDVB nanofiber composites are achieved during polymerization. Emulsification of the oleic acid-capped $\mathrm{Fe}_{3} \mathrm{O}_{4}$ NPs and the extrusion force of the internal initiator droplets caused by polymerization facilitate the dispersion of BFEE nano-droplets into smaller droplets. The diameter of the obtained PDVB nanofibers is $50 \mathrm{~nm}$ (Fig. 1a). Through ultrasonic disruption and magnetic separation (Fig. 1b), we can obtain pure ultrafine PDVB nanofibers from the composites.

We were inspired by the "template-free method"16,17 and "nanoseeding,"18-20 for the synthesis of ultrafine PDVB nanofibers. A very small amount of oleic acid-capped $\mathrm{Fe}_{3} \mathrm{O}_{4} \mathrm{NPs}(0.05 \mathrm{wt} \%)$ is added in $n$-hexane, followed by the addition of the initiator BFEE and the monomer DVB. The PDVB ultrafine nanofiber composites are precipitated and the diameter of the new PDVB nanofibers is below $50 \mathrm{~nm}$ (Fig. 2c-e). In comparison, the diameter of our previous nanofibers was 150-200 nm (Fig. 2a and b). ${ }^{8}$

We investigated the effect of different amounts of oleic acidcapped $\mathrm{Fe}_{3} \mathrm{O}_{4}$ NPs on the diameter and morphology of the PDVB nanofibers using SEM (Fig. 3) and TEM (Fig S2, ESI $\dagger$ ). In the presence of a smaller concentration of $\mathrm{Fe}_{3} \mathrm{O}_{4} \mathrm{NPs}(0.01 \mathrm{wt} \%$; $0.02 \mathrm{wt} \%$ ), we can obtain blended PDVB nanofibers with a diameter of about $200 \mathrm{~nm}$ (coarse) and below $50 \mathrm{~nm}$ (ultrafine) (Fig. $3 \mathrm{a}$ and b). With an increase in the $\mathrm{Fe}_{3} \mathrm{O}_{4} \mathrm{NP}$ content, the proportion of ultrafine PDVB nanofibers gradually increases. When the $\mathrm{Fe}_{3} \mathrm{O}_{4} \mathrm{NP}$ content is $0.05 \mathrm{wt} \%$, the diameter of all of the PDVB nanofibers is under $50 \mathrm{~nm}$ (Fig. 2c). With a further 


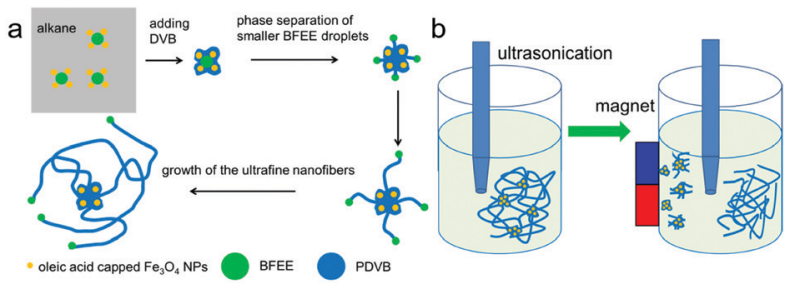

Fig. 1 (a) Schematic of the formation and growth of ultrafine PDVB nanofiber composites; and (b) schematic of the ultrasonic disruption and magnetic separation of ultrafine PDVB nanofibers.

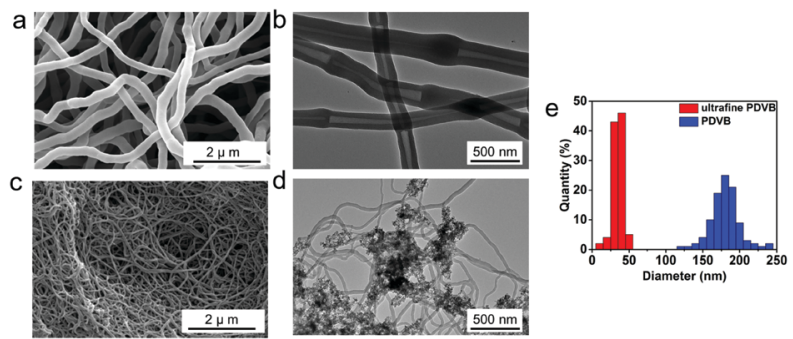

Fig. 2 Comparison of PDVB nanofibers with ultrafine PDVB nanofiber composites. (a) SEM image and (b) TEM image of PDVB nanofibers synthesized without $\mathrm{Fe}_{3} \mathrm{O}_{4} \mathrm{NPs}$. (c) SEM image and (d) TEM image of ultrafine PDVB nanofiber composites synthesized at 0.05 wt\% of $\mathrm{Fe}_{3} \mathrm{O}_{4}$ NPs. DVB and BFEE are fixed at 2 wt\% and 0.05 wt\%. (e) Diameter distribution of PDVB nanofibers and ultrafine PDVB nanofibers.
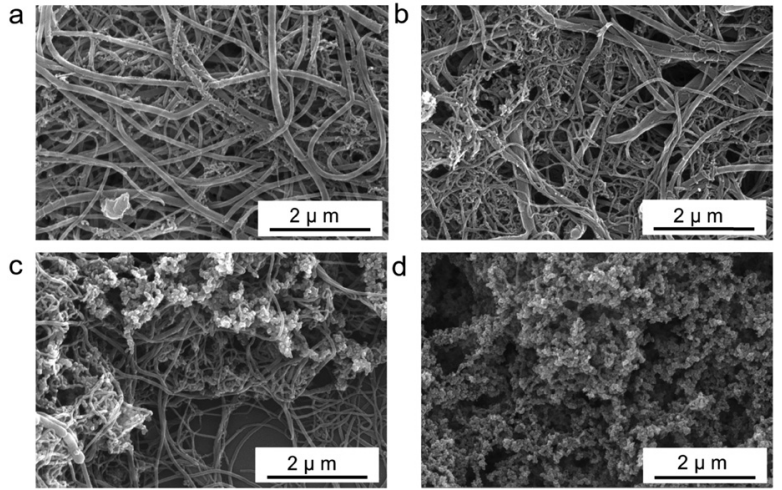

Fig. 3 SEM images of PDVB nanofibers synthesized at different concentration of $\mathrm{Fe}_{3} \mathrm{O}_{4} \mathrm{NPs}$ : (a) 0.01 wt\%; (b) 0.02 wt\%; (c) 0.1 wt\%; and (d) $0.2 \mathrm{wt} \%$. DVB and BFEE are fixed at $2 \mathrm{wt} \%$ and $0.05 \mathrm{wt} \%$.

increase in the $\mathrm{Fe}_{3} \mathrm{O}_{4} \mathrm{NP}$ content, the product obtained is ultrafine PDVB nanofibers mixed with irregular protrusions (Fig. 3c). The proportion of irregular protrusions increases as the amount of $\mathrm{Fe}_{3} \mathrm{O}_{4} \mathrm{NP}$ is increased. When the $\mathrm{Fe}_{3} \mathrm{O}_{4} \mathrm{NP}$ content reaches $0.2 \mathrm{wt} \%$, the products become irregular protrusions. The diameter of these protrusions is about $30 \mathrm{~nm}$ and the protrusions are easily agglomerated to form coral-like structures (Fig. 3d). In addition, in the case of a low $\mathrm{Fe}_{3} \mathrm{O}_{4} \mathrm{NP}$ content (below $0.02 \mathrm{wt} \%$ ), there are also irregular protruding structure exists, although their content is low (Fig S3, ESI $\dagger$ ).

As the reaction progresses, the morphology of the composites changes (Fig. 4 and Fig. S4, ESI $\dagger$ ), and we explain the mechanism of the evolution of the ultrafine nanofiber composite as follows (Fig. 1a). The oleic acid on the surface of the $\mathrm{Fe}_{3} \mathrm{O}_{4}$ NPs acts as an emulsifier for the BFEE and the $n$-hexane, so that the $\mathrm{Fe}_{3} \mathrm{O}_{4}$ NPs tend to distribute on the surface of the BFEE droplets and the BFEE droplets are dispersed into the smaller BFEE droplets. After adding DVB, the cationic polymerization of the monomer initially starts on the surface of the BFEE droplets, thereby forming cationic living polymer chains of PDVB. When the cationic living polymer chains meet the oleic acid-capped $\mathrm{Fe}_{3} \mathrm{O}_{4}$ NPs, they reacted with the reactive double bonds of the oleic acid. ${ }^{21}$ The oleic acid-capped $\mathrm{Fe}_{3} \mathrm{O}_{4}$ NPs are encapsulated into PDVB forming $\mathrm{Fe}_{3} \mathrm{O}_{4} \mathrm{NP} / \mathrm{PDVB}$ core-shell composite nanoparticles. The nanoscale composites are further polymerized into aggregates and dendrites (Fig. 4a). PDVB further polymerizes and squeezes the BFEE inside, causing the phase separation of BFEE, and forming smaller BFEE nanoscale droplets (Fig. 1a). Ultrafine PDVB nanofibers are grown from surface of the aggregates (Fig. $4 \mathrm{~b}$ and c). With the movement of the BFEE nanoscale droplets, the ultrafine PDVB nanofibers gradually grow longer and are interwoven (Fig. 4d).

We can separate pure ultrafine PDVB nanofibers from the magnetic dendrites using the method of ultrasonic disruption and magnetic separation (Fig. 1b). We disperse the magnetic PDVB nanofiber composites (Fig. 5a) into ethanol and break them using an ultrasonic cell homogenizer for more than 20 minutes. Part of the ultrafine nanofibers can be broken and separated from the irregular magnetic dendrites that encapsulate the $\mathrm{Fe}_{3} \mathrm{O}_{4}$ NPs. When we use the magnet to attract the composites during the entire ultrasonic process, the $\mathrm{Fe}_{3} \mathrm{O}_{4}$ NPs-enriched dendrites part is attracted more closely to the magnet (Fig. 5b), so we can obtain a higher proportion of ultrafine PDVB nanofibers at the farthest position from the magnet (Fig. 5c). The length of separated nanofibers is about 10-15 $\mu \mathrm{m}$, but the proportion of magnetic composite in the separated nanofibers is significantly reduced. We can obtain purer ultrafine PDVB nanofibers when extending the time close to 40 minutes of ultrasonic disruption, but the length of the separated
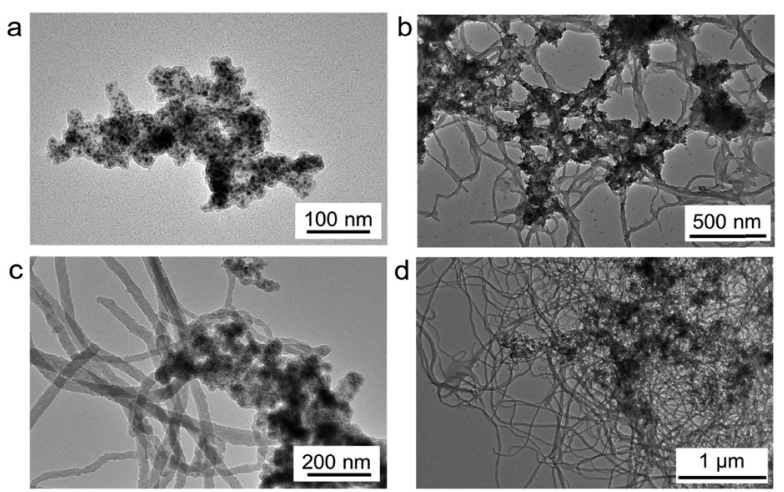

Fig. 4 TEM images of the morphological evolution of the composites with different polymerization times. (a) Irregular aggregates; ( $b$ and $c$ ) ultrafine PDVB nanofibers growing from the aggregate surface; and (d) the aggregates and the ultrafine nanofibers interwoven forming a network. DVB, BFEE and the oleic acid-capped $\mathrm{Fe}_{3} \mathrm{O}_{4} \mathrm{NPs}$ are fixed at $2 \mathrm{wt} \%$, $0.05 w t \%$ and $0.05 w t \%$, respectively. 

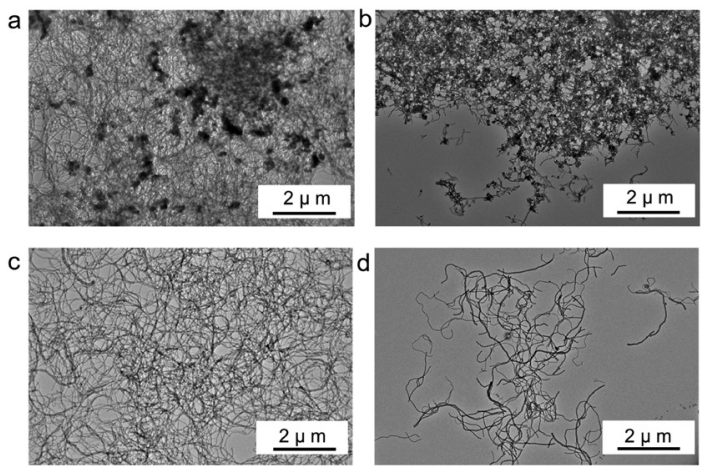

Fig. 5 Ultrafine PDVB nanofibers are separated through ultrasonic disruption and magnetic separation of the composites: (a) TEM image of ultrafine PDVB nanofiber composites synthesized with $\mathrm{Fe}_{3} \mathrm{O}_{4} \mathrm{NPs}$ before ultrasonic disruption; (b) TEM image of the $\mathrm{Fe}_{3} \mathrm{O}_{4} \mathrm{NP}$-enriched part of the composites attracted with a magnet after ultrasonic disruption; (c) TEM image of the ultrafine PDVB nanofiber-enriched part; and (d) TEM image of shorter ultrafine PDVB nanofibers when extending the ultrasonic disruption time.

ultrafine PDVB nanofibers is broken to lengths below $5 \mu \mathrm{m}$ (Fig. $5 \mathrm{~d}$ ). Furthermore, the oleic acid on the $\mathrm{Fe}_{3} \mathrm{O}_{4}$ NPs acts as an emulsifier and plays an important role in the further dispersion of the BFEE nano-droplets; however, only using surfactant (such as oleic acid or its derivatives) during the synthesis, it is difficult to obtain pure ultrafine nanofibers, ${ }^{21}$ even by means of separation.

Furthermore, there are a few reports on the use of templatefree polymerization to synthesize polymer nanofibers successfully, except for polyaniline, ${ }^{18}$ polypyrrole $^{22}$ and PDVB. The polymerization of polyaniline and polypyrrole nanofibers is an oxidation reaction, while the polymerization of PDVB nanofibers is a cationic polymerization. Compared with polyaniline and polypyrrole nanofibers, PDVB nanofibers have abundant reactive groups, which are a great advantage for further modification if they are to be used in nanomaterials and nanodevices. For a polymer to be synthesized using a template-free method of forming nanofibers, the combination of suitable solvents, initiators, and dopants or surfactants is the key; also in addition, there are still other unknown factors that remain to be discovered. Preparing polymer nanofibers using a templatefree method has the advantages of low cost, high efficiency and ease of large-scale preparation. The template-free synthesis method for nanofibers of other materials needs to be developed.

\section{Conclusions}

In conclusion, we have successfully reduced the diameter of PDVB nanofibers from $200 \mathrm{~nm}$ to less than $50 \mathrm{~nm}$ by adding very small amounts of oleic acid-capped $\mathrm{Fe}_{3} \mathrm{O}_{4}$ NPs during the polymerization of DVB. And through ultrasonic disruption and magnetic separation of the ultrafine PDVB nanofiber composites, we can obtain purified ultrafine PDVB nanofibers without the $\mathrm{Fe}_{3} \mathrm{O}_{4}$ NPs. Moreover, the ultrafine PDVB nanofibers can be produced on a large scale with a high concentration more than $10 \mathrm{wt} \%$ of DVB. Abundant the reactive groups of PVDF nanofibers provide the great advantage of further modification. The ultrafine PDVB nanofibers have great potential applications in nanomaterials and nanodevices with small size requirements.

\section{Conflicts of interest}

There are no conflicts to declare.

\section{Acknowledgements}

This work was supported by the National Natural Science Foundation of China (51833005, T2125003 and 61875015).

\section{Notes and references}

1 Y. Zhao, H. Fu, A. Peng, Y. Ma, D. Xiao and J. Yao, Adv. Mater., 2008, 20, 2859-2876.

2 M. F. De Volder, S. H. Baughman, R. H. Baughman and A. J. Hart, Science, 2013, 339, 535-539.

3 M. Kaempgen, C. K. Chan, J. Ma, Y. Cui and G. Gruner, Nano Lett., 2009, 9, 1872-1876.

4 K. Kostarelos, A. Bianco and M. Prato, Nat. Nanotechnol., 2009, 4, 627-633.

5 H. Bi, Z. Yin, X. Cao, X. Xie, C. Tan, X. Huang, B. Chen, F. Chen, Q. Yang, X. Bu, X. Lu, L. Sun and H. Zhang, Adv. Mater., 2013, 25, 5916-5921.

6 M. H. Dehghani, M. M. Taher, A. K. Bajpai, B. Heibati, I. Tyagi, M. Asif, S. Agarwal and V. Gupta, Chem. Eng. J., 2015, 279, 344-352.

7 Q. Wu, Y. Xu, Z. Yao, A. Liu and G. Shi, ACS Nano, 2010, 4, 1963-1970.

8 W. Ni, F. Liang, J. Liu, X. Qu, C. Zhang, J. Li, Q. Wang and Z. Yang, Chem. Commun., 2011, 47, 4727-4729.

9 D. Lv, W. Ni, F. Liang, Q. Wang, X. Qu and Z. Yang, Chin. J. Polym. Sci., 2015, 33, 1344-1350.

10 S. Cui, X. Ji, F. Liang and Z. Yang, Chin. Chem. Lett., 2015, 26, 942-945.

11 Z. Liu, Q. Wang, F. Liang, X. Qu, C. Zhang, J. Li and Z. Yang, Polymer, 2013, 54, 4948-4954.

12 D. Li and Y. Xia, Adv. Mater., 2004, 16, 1151-1170.

13 Y. Zhao, X. Cao and L. Jiang, J. Am. Chem. Soc., 2007, 129, 764-765.

14 C. R. Martin, Acc. Chem. Res., 1995, 28, 61-68.

15 Q. Zhang, C. Deng, F. Soyekwo, Q. Liu and A. Zhu, Adv. Funct. Mater., 2016, 26, 792-800.

16 X. Zhang and S. K. Manohar, Chem. Commun., 2004, 2360-2361.

17 H. Ding, J. Shen, M. Wan and Z. Chen, Macromol. Chem. Phys., 2008, 209, 864-871.

18 Z. Wei, M. Wan, T. Lin and L. Dai, Adv. Mater., 2003, 15, 136-139.

19 Z. Niu, J. Liu, A. Lee, M. Bruckman, D. Zhao, G. Koley and Q. Wang, Nano Lett., 2007, 7, 3729-3733.

20 X. Zhang, W. Goux and S. Manohar, J. Am. Chem. Soc., 2004, 126, 4502-4503.

21 D. Lv, L. Sheng, J. Wan, J. Dong, H. Ouyang, H. Jiao and J. Liu, Polym. Chem., 2019, 10, 331-335.

22 H. D. Tran, K. Shin, W. G. Hong, J. M. D’Arcy, R. W. Kojima, B. H. Weiller and R. B. Kaner, Macromol. Rapid Commun., 2007, 28, 2289-2293. 